\title{
ON A BERNOULLI-TYPE OVERDETERMINED FREE BOUNDARY PROBLEM
}

\author{
Murat Akman, Agnid Banerjee and Mariana Smit Vega Garcia
}

University of Essex, Department of Mathematical Sciences

Wivenhoe Park, Colchester, Essex CO4 3SQ, United Kingdom; murat.akman@essex.ac.uk

Tata Institute of Fundamental Research, Center for Applicable Mathematics

Bangalore-560065, India; agnidban@gmail.com

Western Washington University, Department of Mathematics

BH230, Bellingham, WA 98225, U.S.A.; Mariana.SmitVegaGarcia@wwu.edu

\begin{abstract}
In this article we study a Bernoulli-type free boundary problem and generalize a work of Henrot and Shahgholian in [25] to $\mathcal{A}$-harmonic PDEs. These are quasi-linear elliptic PDEs whose structure is modelled on the $p$-Laplace equation for a fixed $1<p<\infty$. In particular, we show that if $K$ is a bounded convex set satisfying the interior ball condition and $c>0$ is a given constant, then there exists a unique convex domain $\Omega$ with $K \subset \Omega$ and a function $u$ which is $\mathcal{A}$-harmonic in $\Omega \backslash K$, has continuous boundary values 1 on $\partial K$ and 0 on $\partial \Omega$, such that $|\nabla u|=c$ on $\partial \Omega$. Moreover, $\partial \Omega$ is $C^{1, \gamma}$ for some $\gamma>0$, and it is smooth provided $\mathcal{A}$ is smooth in $\mathbf{R}^{n} \backslash\{0\}$. We also show that the super level sets $\{u>t\}$ are convex for $t \in(0,1)$.
\end{abstract}

\section{Introduction and statement of main results}

Classical Bernoulli free-boundary problems arise in electrostatics, fluid dynamics, optimal insulation, and electro chemistry. In the case of electrostatics, the task is to design an annular condenser consisting of a prescribed boundary component $\partial E$, and an unknown boundary component $\partial \Omega$ (where $\Omega \subset E$ ), such that the electric field $\nabla u$ is constant in magnitude on the surface $\partial \Omega$ of the second conductor (see $[18,19]$ for treatment of this problem and applications). This leads to the existence of the following interior Bernoulli free-boundary (will be denoted by (IBFB)) problem:

$$
(\text { IBFB }) \begin{cases}-\Delta u=0 & \text { in } E \backslash \bar{\Omega}, \\ u=1 & \text { on } \partial E, \\ u=0 & \text { on } \partial \Omega, \\ |\nabla u|=a & \text { on } \partial \Omega\end{cases}
$$

for some given constant $a>0$. The constraint $|\nabla u|=a$ is called Bernoulli's law.

Here Bernoulli's law $|\nabla u|=a$ should be understood in the following sense:

$$
\liminf _{y \rightarrow x, y \in E \backslash \bar{\Omega}}|\nabla u(y)|=\limsup _{y \rightarrow x, y \in E \backslash \bar{\Omega}}|\nabla u(y)|=a \quad \text { for every } \quad x \in \partial \Omega .
$$

The existence and uniqueness of this problem can be stated in the following manner: is there a domain $\Omega$ with $\Omega \subset E$ and a potential $u: E \backslash \Omega \rightarrow \mathbf{R}$ satisfying (1.2)? If so, is the couple $(\Omega, u)$ unique?

https://doi.org/10.5186/aasfm.2021.4639

2020 Mathematics Subject Classification: Primary 35R35, 35A01, 35J92, 35J25, 35J70.

Key words: Quasilinear elliptic equations and $p$-Laplacian, degenerate elliptic equations, free boundary problems, Bernoulli-type free boundary problems.

A. B. is supported in part by SERB Matrix grant MTR/2018/000267 and by Department of Atomic Energy, Government of India, under project no. 12-R \& D-TFR-5.01-0520. 
The exterior Bernoulli free-boundary problem ((EBFB) problem for short) is defined in a similar fashion: is there a couple $(\Omega, u)$ such that $E \subset \Omega$ and (1.2) below holds?

$$
(\text { EBFB }) \begin{cases}-\Delta u=0 & \text { in } \Omega \backslash \bar{E} \\ u=1 & \text { on } \partial E \\ u=0 & \text { on } \partial \Omega, \\ |\nabla u|=a & \text { on } \partial \Omega .\end{cases}
$$

In this paper our main goal is to generalize the work of [25] on the (EBFB) for $\mathcal{A}$ harmonic PDEs (see the definition below in (1.6)), proving existence and uniqueness of $(\Omega, u)$ and showing that $\partial \Omega$ is smooth. (see Theorem 1.4).

Regarding the existing literature, the existence of solutions to the (EBFB) problem was obtained by Alt and Caffarelli in [9] by variational methods, and by Beurling [12] using sub-super solution methods in the plane. The reader is also referred to results of Acker in $[2,1]$ concerning the uniqueness and monotonicity of this problem.

If we assume $E$ to be convex and require $\Omega$ to be convex as well, the question of existence and uniqueness of a pair satisfying (1.2) in the plane was answered affirmatively by Tepper in [35], by Hamilton in [22] both by using conformal mappings, and by Kawohl in [27], using different methods. In higher dimensions, convexity and uniqueness of $\Omega$ were shown by Henrot and Shahgholian in [24]. Under a convexity assumption on $E$, the (EBFB) problem was also studied by Henrot and Shahgholian in [25], where they proved existence of a pair $(\Omega, u)$ satisfying (1.2) without assuming $E$ to be bounded or regular. When $E$ is bounded, it was shown in [25] that the (EBFB) problem has a unique solution, and the same result was obtained independently by Acker and Meyer in [3].

Neither existence nor uniqueness is always true in the (IBFB) case. In the plane, existence of a pair $(\Omega, u)$ satisfying (1.1) was obtained by Lavrentèv in [30], Beurling in [12], and Daniljuk in [14]. A higher dimensional result was proved by Alt and Caffarelli in [9], and under certain assumptions Henrot and Shahgholian proved in [24] that the mean curvature of $\partial \Omega$ is positive for any connected component. In [24], it was shown that if the (IBFB) problem admits a solution and $E$ is convex, then $\Omega$ is also convex.

For further discussion of the problems we consider, we shall first introduce the p-Laplace equation:

$$
\Delta_{p} u=\nabla \cdot\left(|\nabla u|^{p-2} \nabla u\right)=|\nabla u|^{p-4}\left[|\nabla u|^{2} \Delta u+(p-2) \sum_{i, j=1}^{n} u_{x_{i}} u_{x_{j}} u_{x_{i} x_{j}}\right] .
$$

Here $p$ is fixed with $1<p<\infty,|\nabla u|=\left(u_{x_{1}}^{2}+\ldots+u_{x_{n}}^{2}\right)^{1 / 2}$, and $\nabla \cdot$ is the divergence operator.

It is well-known that, in general, solutions of the p-Laplace equation do not enjoy second order derivatives in the classical sense, therefore solutions to these equations have to be understood as weak solutions. That is, given a bounded, connected open set $\Omega \subset \mathbf{R}^{n}, u$ is a $p$-harmonic function in $\Omega$ provided $u>0$ in $\Omega$ and $u$ is in the Sobolev space $W^{1, p}(U)$ for each open set $U$ with $\bar{U} \subset \Omega$ and

$$
\int_{U}|\nabla u|^{p-2}\langle\nabla u, \nabla \eta\rangle \mathrm{d} x=0 \quad \text { whenever } \quad \eta \in W_{0}^{1, p}(U) .
$$


In the above paragraph $W^{1, p}(U)$ denotes the space of equivalence classes of functions $h$ with distributional gradient $\nabla h$ both of which are $p$ integrable in $U$, and $W_{0}^{1, p}$ denotes the closure of $C_{0}^{\infty}$ in the $W^{1, p}$ norm.

In [26], the $p$-Laplace operator was treated in the (IBFB) case:

$$
\begin{cases}\Delta_{p} u=0 & \text { in } E \backslash \Omega \\ u=1 & \text { on } \partial E \\ u=0 & \text { on } \partial \Omega \\ |\nabla u|=a & \text { on } \partial \Omega\end{cases}
$$

Existence of a solution, and regularity (that is, $\partial \Omega$ is $C^{2, \alpha}$ ) were shown in that article.

The authors of [24] proved uniqueness and convexity of the (EBFB) problem if the Laplace operator is replaced by a general nonlinear operator $\mathcal{L}$ of the form $\mathcal{L}=\mathcal{L} u=\mathcal{F}\left(x, u, \nabla u, \nabla^{2} u\right)$, if the operator $\mathcal{L}$ satisfies certain properties (see section 4 in that article).

In this article we consider the (EBFB) problem when the underlying PDE is the so called $\mathcal{A}$-harmonic PDE. We introduce this nonlinear elliptic equation in what follows.

Definition 1.1. Let $p, \alpha \in(1, \infty)$ be fixed and

$$
\mathcal{A}=\left(\mathcal{A}_{1}, \ldots, \mathcal{A}_{n}\right): \mathbf{R}^{n} \backslash\{0\} \rightarrow \mathbf{R}^{n},
$$

be such that $\mathcal{A}=\mathcal{A}(\eta)$ has continuous partial derivatives in $\eta_{k}$ for $k=1,2, \ldots, n$ on $\mathbf{R}^{n} \backslash\{0\}$. We say that the function $\mathcal{A}$ belongs to the class $M_{p}(\alpha)$ if the following two conditions are satisfied whenever $\xi \in \mathbf{R}^{n}$ and $\eta \in \mathbf{R}^{n} \backslash\{0\}$ :

(i) $\alpha^{-1}|\eta|^{p-2}|\xi|^{2} \leq \sum_{i, j=1}^{n} \frac{\partial \mathcal{A}_{i}}{\partial \eta_{j}}(\eta) \xi_{i} \xi_{j} \leq \alpha|\eta|^{p-2}|\xi|^{2}$,

(ii) $\mathcal{A}$ is $p-1$ homogeneous, i.e., $\mathcal{A}(\eta)=|\eta|^{p-1} \mathcal{A}(\eta /|\eta|)$.

We set $\mathcal{A}(0)=0$ and note that Definition 1.1 (i) and (ii) imply

$$
\begin{aligned}
c^{-1}\left(|\eta|+\left|\eta^{\prime}\right|\right)^{p-2}\left|\eta-\eta^{\prime}\right|^{2} & \leq\left\langle\mathcal{A}(\eta)-\mathcal{A}\left(\eta^{\prime}\right), \eta-\eta^{\prime}\right\rangle \\
& \leq c\left|\eta-\eta^{\prime}\right|^{2}\left(|\eta|+\left|\eta^{\prime}\right|\right)^{p-2}
\end{aligned}
$$

whenever $\eta, \eta^{\prime} \in \mathbf{R}^{n} \backslash\{0\}$. We will additionally assume that there exists $1 \leq \Lambda<\infty$ such that

$$
\left|\frac{\partial \mathcal{A}_{i}}{\partial \eta_{j}}(\eta)-\frac{\partial \mathcal{A}_{i}}{\partial \eta_{j}}\left(\eta^{\prime}\right)\right| \leq \Lambda\left|\eta-\eta^{\prime}\right||\eta|^{p-3}
$$

whenever $0<|\eta| \leq 2\left|\eta^{\prime}\right|$ and $1 \leq i, j \leq n$.

Definition 1.2. Given an open set $\Omega \subset \mathbf{R}^{n}$ and $\mathcal{A} \in M_{p}(\alpha)$, one says that $u$ is $\mathcal{A}$-harmonic in $\Omega$, and we write $\nabla \cdot \mathcal{A}(\nabla u)=0$, provided $u>0$ in $\Omega, u \in W^{1, p}(U)$ for each open set $U$ with $\bar{U} \subset \Omega$ and

$$
\int\langle\mathcal{A}(\nabla u(x)), \nabla \eta(x)\rangle \mathrm{d} x=0 \quad \text { whenever } \quad \eta \in W_{0}^{1, p}(U) .
$$

For more about PDEs of this type the reader is referred to [23]. Notice that when $\mathcal{A}(\eta)=\eta$ then (1.6) is the usual Laplace's equation, and when $\mathcal{A}(\eta)=|\eta|^{p-2} \eta$ then (1.6) becomes the $p$-Laplace equation. 
Definition 1.3. We say that a set $K$ satisfies the interior ball condition if

$$
\text { for each } x_{0} \in \partial D \text {, there is a ball } B(z, \delta) \subset D \text { with } x_{0} \in \overline{B(z, \delta)}
$$

for some $\delta>0$.

Our main goal is to generalize the work of Henrot and Shahgholian in [25] on the (EBFB) problem to $\mathcal{A}$-harmonic PDEs. In particular, we show that

Theorem 1.4. Let $c>0$ be a given constant and $K$ be a bounded convex domain satisfying the interior ball condition. Then there exist a unique convex domain $\Omega$ with $K \subset \Omega$ and function $u$ satisfying

$$
\left\{\begin{array}{l}
\nabla \cdot \mathcal{A}(\nabla u)=0 \text { in } \Omega \backslash \bar{K} \\
u \text { has continuous boundary values } 1 \text { on } \partial K \text { and } 0 \text { on } \partial \Omega, \\
\text { the superlevel sets }\{u>t\} \text { are convex for every } t \in(0,1), \\
|\nabla u|=c \text { on } \partial \Omega
\end{array}\right.
$$

Moreover, $\partial \Omega \in C^{1, \gamma}$ for some $\gamma>0$. Furthermore we have that $\partial \Omega$ is smooth provided $\mathcal{A}$ is smooth.

The plan of the paper is as follows. In section 2, we gather some known results concerning the regularity of $\mathcal{A}$-harmonic functions that are relevant for our work. We also show that the levels of $u$ are convex if $K$ is convex by adapting an idea of Lewis [31]. In section 3, using a method of Beurling (see also [25]), we prove the existence result in Theorem 1.4. Uniqueness in Theorem 1.4 will essentially follow from [24]. Finally the regularity result in Theorem 1.4 is obtained using ideas inspired by the work of Vogel in [37].

\section{Notation and preparatory lemmas}

Let $x=\left(x_{1}, \ldots, x_{n}\right)$ denote points in $\mathbf{R}^{n}$ and let $\bar{E}, \partial E$, be the closure and boundary of the set $E \subset \mathbf{R}^{n}$. Let $\langle\cdot, \cdot\rangle$ be the usual inner product in $\mathbf{R}^{n}$ and $|x|^{2}=$ $\langle x, x\rangle$. Let $d(E, F)$ denote the distance between the sets $E$ and $F$. Let $B(x, r)$ be the open ball centered at $x$ with radius $r>0$ in $\mathbf{R}^{n}$ and $d x$ denote the Lebesgue $n$-measure in $\mathbf{R}^{n}$. Given $U \subset \mathbf{R}^{n}$ an open set and $q$ with $1 \leq q \leq \infty$, let $W^{1, q}(U)$ denote equivalence classes of functions $h: \mathbf{R}^{n} \rightarrow \mathbf{R}$ with distributional gradient $\nabla h=$ $\left\langle h_{x_{1}}, \ldots, h_{x_{n}}\right\rangle$, both of which are $q$-integrable in $U$ with Sobolev norm

$$
\|h\|_{W^{1, q}(U)}^{q}=\int_{U}\left(|h|^{q}+|\nabla h|^{q}\right) \mathrm{d} x .
$$

Let $C_{0}^{\infty}(U)$ be the set of infinitely differentiable functions with compact support in $U$ and let $W_{0}^{1, q}(U)$ be the closure of $C_{0}^{\infty}(U)$ in the norm of $W^{1, q}(U)$.

In the sequel, $c$ will denote a positive constant $\geq 1$ (not necessarily the same at each occurrence), which may depend only on $p, n, \alpha, \Lambda$ unless otherwise stated. In general, $c\left(a_{1}, \ldots, a_{n}\right)$ denotes a positive constant $\geq 1$ which may depend only on $p, n, \alpha, \Lambda, a_{1}, \ldots, a_{n}$, which is not necessarily the same at each occurrence. By $A \approx B$ we mean that $A / B$ is bounded above and below by positive constants depending only on $p, n, \alpha, \Lambda$. Finally, in this section we will always assume that $1<p<\infty$, and $r>0$.

We next introduce the notion of the Hausdorff measure. To this end, let $\hat{r}_{0}>0$ be given, and let $0<\delta<\hat{r}_{0}$ be fixed. Let $\operatorname{diam}(\cdot)$ denote the diameter of a set 
and let $E \subseteq \mathbf{R}^{n}$ be a given Borel set. For an arbitrary integer $k>0$, we define the $(\delta, k)$-Hausdorff content of $E$ in the usual way:

$$
\mathcal{H}_{\delta}^{k}(E):=\inf \left\{\sum_{i} r_{i}^{k}: E \subset \bigcup_{i} B\left(x_{i}, r_{i}\right) \text { with } r_{i}<\delta\right\} .
$$

Here the infimum is taken over all possible covers $\left\{B\left(x_{i}, r_{i}\right)\right\}$ of $E$. Then the Hausdorff $k$-measure of $E$ is defined by

$$
\mathcal{H}^{k}(E):=\lim _{\delta \rightarrow 0} \mathcal{H}_{\delta}^{k}(E) .
$$

Lemma 2.1. Given $p$ with $1<p<\infty$, assume that $\mathcal{A} \in M_{p}(\alpha)$ for some $\alpha>1$. Let $u$ be an $\mathcal{A}$-harmonic function in $B(w, 4 r)$. Then

(a) $r^{p-n} \int_{B(w, r / 2)}|\nabla u|^{p} \mathrm{~d} x \leq c \max _{B(w, r)} u^{p}$,

(b) $\max _{B(w, r)} u \leq c \min _{B(w, r)} u$.

Moreover, there exists $\gamma \in(0,1)$ depending on $p, n, \alpha$ such that if $x, y \in B(w, r)$, then

(c) $|u(x)-u(y)| \leq c(|x-y| / r)^{\gamma} \max _{B(w, 2 r)} u$.

For a proof of Lemma 2.1 see [34].

Lemma 2.2. Given $p$ with $1<p<\infty$, assume that $\mathcal{A} \in M_{p}(\alpha)$ for some $\alpha>1$. Let $u$ be an $\mathcal{A}$-harmonic function in $B(w, 4 r)$. Then $u$ has a representative locally in $W^{1, p}(B(w, 4 r))$ with Hölder continuous partial derivatives in $B(w, 4 r)$ (also denoted $u)$, and there exist $\beta \in(0,1]$ and $c \geq 1$ depending only on $p, n, \alpha$ such that if $x, y \in B(w, r)$, then

(i) $|\nabla u(x)-\nabla u(y)| \leq c(|x-y| / r)^{\beta} \max _{B(w, r)}|\nabla u| \leq c r^{-1}(|x-y| / r)^{\beta} u(w)$.

(ii) $\int_{B(w, r)} \sum_{i, j=1}^{n}|\nabla u|^{p-2}\left|u_{x_{i} x_{j}}\right|^{2} \mathrm{~d} x \leq c r^{n-p-2} u(w)$.

Moreover, if

$$
\gamma r^{-1} u \leq|\nabla u| \leq \gamma^{-1} r^{-1} u \quad \text { on } B(w, 2 r)
$$

for some $\gamma \in(0,1)$ and (1.5) holds then $u$ has Hölder continuous second partial derivatives in $B(w, r)$ and there exists $\theta \in(0,1), \bar{c} \geq 1$, depending only on the data and $\gamma$ such that if $x, y \in B(w, r / 2)$, then

$$
\begin{aligned}
{\left[\sum_{i, j=1}^{n}\left(u_{x_{i} x_{j}}(x)-u_{y_{i} y_{j}}(y)\right)^{2}\right]^{1 / 2} } & \leq \bar{c}(|x-y| / r)^{\theta} \max _{B(w, r)}\left(\sum_{i, j=1}^{n}\left|u_{x_{i} x_{j}}\right|\right) \\
& \leq \bar{c}^{2} r^{n}(|x-y| / r)^{\theta}\left(\sum_{i, j=1}^{n} \int_{B(w, 2 r)} u_{x_{i} x_{j}}^{2} r d x\right)^{1 / 2} \\
& \leq \bar{c}^{3} r^{-2}(|x-y| / r)^{\theta} u(w) .
\end{aligned}
$$

A proof of (2.2) can be found in [36]. Estimate (2.3) follows from (2.2), the added assumptions and Schauder type estimates (see [21]).

We will make use of following lemma when we rotate our coordinate system. A proof of it can be found in [32, Lemma 2.15]. 
Lemma 2.3. Let $\Omega \subset \mathbf{R}^{n}$ be a domain and let $p$ with $1<p<\infty$ be given. Let $\mathcal{A} \in M_{p}(\alpha)$ for some $\alpha>1$ and $u$ be $\mathcal{A}$-harmonic in $\Omega$. If $F: \mathbf{R}^{n} \rightarrow \mathbf{R}^{n}$ is the composition of a translation and a dilation, then

$$
\hat{u}(z)=u(F(z)) \text { is } \mathcal{A} \text {-harmonic in } F^{-1}(\Omega) .
$$

Moreover, if $\tilde{F}: \mathbf{R}^{n} \rightarrow \mathbf{R}^{n}$ is the composition of a translation, a dilation, and a rotation then

$$
\tilde{u}(z)=u(F(z)) \text { is } \hat{\mathcal{A}} \text {-harmonic in } \tilde{F}^{-1}(\Omega) \text { for some } \hat{\mathcal{A}} \in M_{p}(\alpha) .
$$

In what follows we make observations that will be useful throughout the paper (see also $[7,8]$ for a similar computation). Define

$$
\mathcal{L}(\eta, \xi)=\sum_{i, j=1}^{n} \frac{\partial}{\partial x_{i}}\left[b_{i j}(\eta) \xi_{x_{j}}\right], \quad \text { where } b_{i j}(\eta)=\frac{\partial \mathcal{A}_{i}}{\partial \eta_{j}}(\eta) .
$$

When $\eta=\nabla u$ we will write $\mathcal{L}(\eta, \xi)=\mathcal{L}_{u} \xi$, and when $\xi=\nabla w$ we will write $\mathcal{L}_{u} \xi=$ $\mathcal{L}_{u} w$. We next show that if $u$ is $\mathcal{A}$-harmonic in $\Omega$, then $\xi=u$ and $\xi=u_{x_{k}}$ (for $k=1, \ldots, n)$ are both weak solutions to $\mathcal{L}_{u} \xi=0$.

We first see that if $u$ is $\mathcal{A}$-harmonic then

$$
\mathcal{L}_{u} u=\sum_{i, j=1}^{n} \frac{\partial}{\partial x_{i}}\left[\frac{\partial \mathcal{A}_{i}}{\partial \eta_{j}}(\nabla u) u_{x_{j}}\right]=0 .
$$

Indeed, using the $(p-1)$-homogeneity of $\mathcal{A}$ in Definition 1.1 we obtain

$$
\mathcal{L}_{u} u=(p-1) \sum_{i=1}^{n} \frac{\partial}{\partial x_{i}} \mathcal{A}_{i}(\nabla u)=(p-1) \nabla \cdot \mathcal{A}(\nabla u)=0 .
$$

To show that $\mathcal{L}_{u} u_{x_{k}}=0$ for $k=1, \ldots, n$, using Lemma 2.2 we first get $u \in$ $W^{2,2}(\Omega)$. Then it follows that

$$
\mathcal{L}_{u} u_{x_{k}}=\sum_{i, j=1}^{n} \frac{\partial}{\partial x_{i}}\left[\frac{\partial \mathcal{A}_{i}}{\partial \eta_{j}}(\nabla u) u_{x_{k} x_{j}}\right]=\frac{\partial}{\partial x_{k}} \nabla \cdot \mathcal{A}(\nabla u)=0 .
$$

Note that above argument should be understood in the weak sense. Using these two observations and the structural assumptions on $\mathcal{A}$ from Definition 1.1 we also conclude that

$$
\begin{aligned}
\mathcal{L}_{u}\left(|\nabla u|^{2}\right) & =\sum_{i, j=1}^{n} \frac{\partial}{\partial x_{i}}\left[\frac{\partial \mathcal{A}_{i}}{\partial \eta_{j}}(\nabla u)\left(u_{x_{1}}^{2}+\ldots+u_{x_{n}}^{2}\right)_{x_{j}}\right]=2 \sum_{i, j, k=1}^{n} \frac{\partial}{\partial x_{i}}\left[\frac{\partial \mathcal{A}_{i}}{\partial \eta_{j}}(\nabla u) u_{x_{k}} u_{x_{k} x_{j}}\right] \\
& \stackrel{(2.5)}{=} 2 \sum_{i, j, k=1}^{n} \frac{\partial \mathcal{A}_{i}}{\partial \eta_{j}}(\nabla u) u_{x_{k} x_{i}} u_{x_{k} x_{j}} \geq 2 \alpha^{-1}|\nabla u|^{p-2} \sum_{i, j=1}^{n}\left(u_{x_{i} x_{j}}\right)^{2} .
\end{aligned}
$$

Using this observation we conclude

$$
\mathcal{L}_{u}\left(|\nabla u|^{2}\right) \geq c^{-1}|\nabla u|^{p-2} \sum_{i, j=1}^{n}\left(u_{x_{i} x_{j}}\right)^{2} .
$$

Lemma 2.4. Let $\Omega$ be a domain, $K$ be a bounded, closed, convex set with $K \subset \Omega, 1<p<\infty$ and $\alpha>1$ be given. Let $\mathcal{A} \in M_{p}(\alpha)$ and $u$ be $\mathcal{A}$-harmonic in 
$\Omega \backslash K$ with $u=1$ on $\partial K$. If $K$ satisfies the interior ball property then then there exists $c_{*} \geq 1$, depending only on $p, n, \alpha, r_{0}$ such that if $x \in \Omega \backslash K$

$$
\begin{aligned}
& \text { (a) } c_{*}\langle\nabla u(x), z-x\rangle \geq u(x), \\
& \text { (b) } c_{*}^{-1}|x|^{\frac{1-n}{p-1}} \leq|\nabla u(x)| \leq c_{*}|x|^{\frac{1-n}{p-1}} .
\end{aligned}
$$

Proof. A proof of this lemma can be found in [5] when $1<p<n$ and in [6] when $n \leq p<\infty$. The proof uses Lemmas 2.2, 2.1, and 2.3. A barrier type argument is also used, as in [32, Section 2] and [8, Section 4]. We skip the details.

Now let $u$ be a $\mathcal{A}$-harmonic function in $\Omega \backslash K$ where $K \subset \Omega$, it is continuous on $\mathbf{R}^{n}$ with $u \equiv 1$ on $K$ and $u \equiv 0$ on $\mathbf{R}^{n} \backslash \Omega$. The following lemma establishes the convexity of the superlevel sets $\{u>t\}$ when both $K$ and $\Omega$ are convex. We note that such a result plays a crucial role in the uniqueness assertion in Theorem 1.4. In the case of $p$-Laplacian, such a result was first established by Lewis in [31] following Gabriel's ideas as in [20]. Here we adapt the techniques in [31] and [5] to our situation. We also refer to the interesting paper [13] for a different proof in the case of the Laplacian.

Lemma 2.5. Let $K \subset \Omega$ be such that $K, \Omega$ are convex and let $u$ be $\mathcal{A}$-harmonic in $\Omega \backslash K$, continuous on $\mathbf{R}^{n}$ with $u \equiv 1$ on $K$ and $u \equiv 0$ on $\mathbf{R}^{n} \backslash \Omega$. If $K$ satisfies interior ball condition then for each $t \in(0,1)$, the set $\{x \in \Omega: u(x)>t\}$ is convex.

Proof. We note from (2.7) and Lemma 2.2 that

$$
\left\{\begin{array}{l}
|\nabla u| \neq 0 \\
u \text { has Hölder continuous second partial derivatives on compact subsets } \\
\text { of } \Omega \text {. }
\end{array}\right.
$$

Our proof of Lemma 2.5 is by contradiction, following the proof in [31, section 4]. We define for $\hat{x} \in \mathbf{R}^{n}$,

$$
\begin{aligned}
& \mathbf{u}(\hat{x})=\sup _{\hat{y}, \hat{z} \in \mathbf{R}^{n}} \min \{u(\hat{y}), u(\hat{z})\} \\
& \hat{x}=\lambda \hat{y}+(1-\lambda) \hat{z}, \lambda \in[0,1]
\end{aligned}
$$

Notice that $u \leq \mathbf{u}, \mathbf{u} \equiv 1$ in $K$ and $\mathbf{u} \equiv 0$ in $\mathbf{R}^{n} \backslash \Omega$. It suffices to show that $\mathbf{u}=u$. If that were not true, then from the convexity of $K$, continuity of $u$, and the fact that as $w \rightarrow w_{0} \in \partial \Omega, u(w) \rightarrow 0$, we would conclude that there must exist $\epsilon>0$, and $x_{0} \in \Omega$ such that

$$
0<\mathbf{u}^{1+\epsilon}\left(x_{0}\right)-u\left(x_{0}\right)=\max _{\mathbf{R}^{n}}\left(\mathbf{u}^{1+\epsilon}-u\right) .
$$

For ease of writing we write $\mathbf{v}=\mathbf{u}^{1+\epsilon}$ and $v=u^{1+\epsilon}$. There exist $\lambda \in(0,1)$ and $y_{0}, z_{0} \in \Omega \backslash\left\{x_{0}\right\}$ with

$$
x_{0}=\lambda y_{0}+(1-\lambda) z_{0} \quad \text { and } \quad \mathbf{v}\left(x_{0}\right)=\min \left\{v\left(y_{0}\right), v\left(z_{0}\right)\right\} .
$$

We first show that

$$
v\left(y_{0}\right)=v\left(z_{0}\right) .
$$

Assume for contradiction, for instance, that $v\left(y_{0}\right)<v\left(z_{0}\right)$. Since $u \leq \mathbf{u}, u\left(x_{0}\right) \leq$ $u\left(y_{0}\right)=\mathbf{u}\left(x_{0}\right)<u\left(z_{0}\right)$. By continuity, if $z$ is in a small enough neighborhood of $z_{0}$, then $u(z)>u\left(y_{0}\right)+\left(u\left(z_{0}\right)-u\left(y_{0}\right)\right) / 2$. Since $|\nabla u| \neq 0$ in $\Omega$, we can choose $y^{\prime}$ close enough to $y_{0}$ so that $u\left(y^{\prime}\right)>u\left(y_{0}\right)$ and also such that after connecting $y^{\prime}$ and $x_{0}$ by a line, we can pick a corresponding $z^{\prime}$ in the previous neighborhood of $z_{0}$. In this 
manner $\mathbf{u}\left(x_{0}\right) \geq \min \left\{u\left(y^{\prime}\right), u\left(z^{\prime}\right)\right\}>u\left(y_{0}\right)=\mathbf{u}\left(x_{0}\right)$, a contradiction. Thus (2.11) is true.

Next we prove that

$$
\xi=\frac{\nabla v\left(y_{0}\right)}{\left|\nabla v\left(y_{0}\right)\right|}=\frac{\nabla v\left(z_{0}\right)}{\left|\nabla v\left(z_{0}\right)\right|}=\frac{\nabla u\left(x_{0}\right)}{\left|\nabla u\left(x_{0}\right)\right|}
$$

Indeed, let us show that

$$
\frac{\nabla v\left(y_{0}\right)}{\left|\nabla v\left(y_{0}\right)\right|}=\frac{\nabla v\left(x_{0}\right)}{\left|\nabla v\left(x_{0}\right)\right|}
$$

Let $y$ not be on the line through $y_{0}$ and $z_{0}$ and be such that $\nabla v\left(y_{0}\right) \cdot\left(y-y_{0}\right)>0$. Draw the line through $y$ and $z_{0}$ and denote by $x$ its intersection with the line originating at $x_{0}$ with direction $y-y_{0}$. One has that $v(y)>v\left(y_{0}\right)$ for $y$ close to $y_{0}$. Therefore $\tilde{v}\left(x_{0}\right) \leq \tilde{v}(x)$, for $y$ near $y_{0}$. From (2.9) we conclude $u(x) \geq u\left(x_{0}\right)$, for $y$ close to $y_{0}$, hence $\nabla u\left(x_{0}\right) \cdot\left(y-y_{0}\right) \geq 0$ whenever $\nabla v\left(y_{0}\right) \cdot\left(y-y_{0}\right)>0$, showing that $\nabla u\left(x_{0}\right)$ and $\nabla v\left(y_{0}\right)$ point in the same direction.

To simplify our notation, let

$$
A=\left|\nabla v\left(y_{0}\right)\right|, \quad B=\left|\nabla v\left(z_{0}\right)\right|, \quad C=\left|\nabla u\left(x_{0}\right)\right|, \quad a=\left|x_{0}-y_{0}\right|, \quad b=\left|x_{0}-z_{0}\right| .
$$

Let $\eta \in \mathbf{S}^{n-1}$ be such that $\xi \cdot \eta>0$. From (2.8) we can write

$$
\begin{aligned}
& v\left(y_{0}+\rho \eta\right)=v\left(y_{0}\right)+A_{1} \rho+A_{2} \rho^{2}+o\left(\rho^{2}\right), \\
& v\left(z_{0}+\rho \eta\right)=v\left(z_{0}\right)+B_{1} \rho+B_{2} \rho^{2}+o\left(\rho^{2}\right), \\
& u\left(x_{0}+\rho \eta\right)=u\left(x_{0}\right)+C_{1} \rho+C_{2} \rho^{2}+o\left(\rho^{2}\right)
\end{aligned}
$$

as $\rho \rightarrow 0$. Also

$$
A_{1} / A=B_{1} / B=C_{1} / C=\xi \cdot \eta,
$$

where the coefficients and $o\left(\rho^{2}\right)$ depend on $\eta$. Given $\eta$ with $\xi \cdot \eta>0$ and $\rho_{1}$ sufficiently small we see from (2.8) that the inverse function theorem can be used to obtain $\rho_{2}$ with

We conclude as $\rho_{1} \rightarrow 0$ that

$$
v\left(y_{0}+\frac{\rho_{1}}{A} \eta\right)=v\left(z_{0}+\frac{\rho_{2}}{B} \eta\right) .
$$

$$
\rho_{2}=\rho_{1}+\frac{B}{B_{1}}\left(\frac{A_{2}}{A^{2}}-\frac{B_{2}}{B^{2}}\right) \rho_{1}^{2}+o\left(\rho_{1}^{2}\right) .
$$

Now from geometry we see that $\lambda=\frac{b}{a+b}$ so

$$
x=x_{0}+\eta \frac{\left[\rho_{1} \frac{b}{A}+\rho_{2} \frac{a}{B}\right]}{a+b}=\lambda\left(y_{0}+\frac{\rho_{1}}{A} \eta\right)+(1-\lambda)\left(z_{0}+\frac{\rho_{2}}{B} \eta\right) .
$$

From this equality and Taylor's theorem for second derivatives we have

$$
\begin{aligned}
u(x)-u\left(x_{0}\right)= & C_{1}\left[\rho_{1} \frac{\lambda}{A}+\rho_{2} \frac{(1-\lambda)}{B}\right]+C_{2}\left[\rho_{1} \frac{\lambda}{A}+\rho_{2} \frac{(1-\lambda)}{B}\right]^{2} \\
= & C_{1} \rho_{1} \frac{(1-\lambda) A+\lambda B}{A B}+C_{1} \frac{(1-\lambda)}{B_{1}}\left(\frac{A_{2}}{A^{2}}-\frac{B_{2}}{B^{2}}\right) \rho_{1}^{2} \\
& +C_{2} \rho_{1}^{2}\left(\frac{(1-\lambda) A+\lambda B)}{A B}\right)^{2}+o\left(\rho_{1}^{2}\right) .
\end{aligned}
$$

Now

$$
v\left(y_{0}+\frac{\rho_{1}}{A} \eta\right)-u(x) \leq \mathbf{v}(x)-u(x) \leq \mathbf{v}\left(x_{0}\right)-u\left(x_{0}\right)=v\left(y_{0}\right)-u\left(x_{0}\right) .
$$


Hence the mapping

$$
\rho_{1} \rightarrow v\left(y_{0}+\frac{\rho_{1}}{A} \eta\right)-u(x)
$$

has a maximum at $\rho_{1}=0$. Using the Taylor expansion for $v\left(y_{0}+\frac{\rho_{1}}{A} \eta\right)$ in $(2.13)$ and $u(x)$ in $(2.15)$ we have

$$
\begin{aligned}
v\left(y_{0}+\frac{\rho_{1}}{A} \eta\right)-u(x)= & v\left(y_{0}\right)+\frac{A_{1}}{A} \rho_{1}+\frac{A_{2}}{A^{2}} \rho_{1}^{2}-u\left(x_{0}\right) \\
& -C_{1} \rho_{1} \frac{(1-\lambda) A+\lambda B}{A B}-\frac{C_{1}}{a+b} \frac{a}{B_{1}}\left(\frac{A_{2}}{A^{2}}-\frac{B_{2}}{B^{2}}\right) \rho_{1}^{2} \\
& -C_{2} \rho_{1}^{2}\left(\frac{(1-\lambda) A+\lambda B}{A B}\right)^{2}+o\left(\rho_{1}^{2}\right) .
\end{aligned}
$$

Now from the calculus second derivative test, the coefficient of $\rho_{1}$ should be zero and the coefficient of $\rho_{1}^{2}$ should be non-positive. Hence combining terms we get

$$
\frac{A_{1}}{A}=C_{1} \frac{(1-\lambda) A+\lambda B}{A B}
$$

so taking $\eta=\xi$ we arrive first at

$$
\frac{1}{C}=\frac{(1-\lambda) A+\lambda B}{A B}=\frac{(1-\lambda)}{B}+\frac{\lambda}{A} .
$$

Second using (2.16) in the $\rho_{1}^{2}$ term we find that

$$
0 \geq \frac{A_{2}}{A^{2}}-C_{1} \frac{(1-\lambda)}{B_{1}}\left(\frac{A_{2}}{A^{2}}-\frac{B_{2}}{B^{2}}\right)-\frac{C_{2}}{C^{2}} .
$$

Using $C_{1} / B_{1}=C / B$ and doing some arithmetic in (2.17) we obtain

$$
0 \geq(1-K) \frac{A_{2}}{A^{2}}+K \frac{B_{2}}{B^{2}}-\frac{C_{2}}{C^{2}}
$$

where

$$
K=\frac{(1-\lambda) A}{(1-\lambda) A+\lambda B}<1
$$

We now focus on (2.18) by writing $A_{1}, B_{1}, C_{1}$ in terms of derivatives of $u$ and $v$;

$$
0 \geq \sum_{i, j=1}^{n}\left[\frac{(1-K)}{A^{2}} v_{x_{i} x_{j}}\left(y_{0}\right)+\frac{K}{B^{2}} v_{x_{i} x_{j}}\left(z_{0}\right)-\frac{u_{x_{i} x_{j}}\left(x_{0}\right)}{C^{2}}\right] \eta_{i} \eta_{j} .
$$

From symmetry and continuity considerations we observe that (2.19) holds whenever $\eta \in \mathbf{S}^{n-1}$ Thus if

$$
w(x)=-\frac{(1-K)}{A^{2}} v\left(y_{0}+x\right)-\frac{K}{B^{2}} v\left(z_{0}+x\right)+\frac{u_{x_{i} x_{j}}\left(x_{0}+x\right)}{C^{2}},
$$

then the Hessian matrix of $w$ at $x=0$ is positive semi-definite, i.e, $\left(w_{x_{i} x_{j}}(0)\right)$ has non-negative eigenvalues. Also from (i) of Definition 1.1 we see that if

$$
\left.\left.a_{i j}=\frac{1}{2}\left[\frac{\partial \mathcal{A}_{i}}{\partial \eta_{j}}(\xi)\right)+\frac{\partial \mathcal{A}_{j}}{\partial \eta_{i}}(\xi)\right)\right], \quad 1 \leq i, j \leq n,
$$

then $\left(a_{i j}\right)$ is positive definite. From these two observations we conclude that

$$
\operatorname{trace}\left(\left(\left(a_{i j}\right) \cdot\left(w_{x_{i} x_{j}}(0)\right)\right) \geq 0\right. \text {. }
$$


To obtain a contradiction we observe from (1.6), the divergence theorem, (2.18), and $p-2$ homogeneity of partial derivatives of $\mathcal{A}_{i}$, that

$$
\sum_{i, j=1}^{n} a_{i j} u_{x_{j} x_{i}}=|\nabla u|^{2-p} \sum_{i, j=1}^{n} \frac{\partial \mathcal{A}_{i}}{\partial \eta_{j}}(\nabla u) u_{x_{j} x_{i}}=0 \quad \text { at } x_{0}, y_{0}, z_{0} .
$$

Moreover, from the definition of $v$ we have

$$
\begin{aligned}
& v_{x_{i}}=\left(u^{1+\epsilon}\right)_{x_{i}}=(1+\epsilon) u^{\epsilon} u_{x_{i}}, \\
& v_{x_{i} x_{j}}=(1+\epsilon) \epsilon u^{\epsilon-1} u_{x_{i}} u_{x_{j}}+(1+\epsilon) u^{\epsilon} u_{x_{i} x_{j}} .
\end{aligned}
$$

Using (2.21), (2.22), we find that

$$
\begin{aligned}
& |\nabla u|^{p-2} \sum_{i, j=1}^{n} a_{i j} v_{x_{j} x_{i}}=\sum_{i, j=1}^{n} \frac{\partial \mathcal{A}_{i}}{\partial \eta_{j}}(\nabla u)\left[(1+\epsilon) u^{\epsilon-1} u_{x_{j}} u_{x_{i}}+(1+\epsilon) u^{\epsilon} u_{x_{j} x_{i}}\right] \\
& =(1+\epsilon) \epsilon u^{\epsilon-1} \sum_{i, j=1}^{n} \frac{\partial \mathcal{A}_{i}}{\partial \eta_{j}}(\nabla u) u_{x_{j}} u_{x_{i}}+(1+\epsilon) u^{\epsilon} \sum_{i, j=1}^{n} \frac{\partial \mathcal{A}_{i}}{\partial \eta_{j}}(\nabla u) u_{x_{j} x_{i}} \\
& \geq \alpha^{-1}(1+\epsilon) \epsilon u^{\epsilon-1}|\nabla u|^{p-2}|\nabla u|^{2}+0>0
\end{aligned}
$$

at points $y_{0}$ and $z_{0}$ ( $\nabla u$ is also evaluated at these points). Using (2.21), (2.23), we conclude that

$$
\operatorname{trace}\left(\left(a_{i j}\right) \cdot\left(w_{x_{i} x_{j}}(0)\right)\right)=\sum_{i, j=1}^{n} a_{i j} w_{x_{i} x_{j}}(0)<0 .
$$

Equations (2.24) and (2.20) contradict each other. The proof of Lemma 2.5 is now complete.

\section{Proof of Theorem 1.4}

In this section we give a proof of Theorem 1.4 by a method of Beurling, inspired by Henrot and Shahgholian in [25]. To this end, Let $K$ be a convex domain and let $P_{x_{0}, a}$ denote the hyperplane in $\mathbf{R}^{n}$ passing through $x_{0}$ with the normal $a \neq 0$ pointing away from $K$.

A supporting hyperplane to $K$ at boundary point $x_{0}$ is a plane satisfying

$$
P_{x, a}:=\left\{x: a^{T} x=a^{T} x_{0}\right\}
$$

where $a \neq 0$ and $a^{T} x \leq a^{T} x_{0}$ for all $x \in K$. By the supporting hyperplane theorem it is known that there exists a supporting hyperplane at every boundary point of a convex set $K$. Let $\Omega$ be another convex set containing $K$.

For each $x \in \partial K$ there exists a point $y_{x} \in \partial \Omega \cap\{z: a \cdot(z-x)>0\}$ satisfying $a \cdot\left(y_{x}-x\right)=\max a \cdot(z-x)$, where maximum is taken over the set $\partial \Omega \cap\{z: a \cdot(z-x)>$ $0\}$.

We will work on convex ring domains. That is, let $D_{1}$ and $D_{2}$ be two convex domains satisfying $D_{1} \subset \overline{D_{1}} \subset D_{2}$. We first need an auxiliary lemma.

Lemma 3.1. Let $D_{1}, D_{2}$ be two convex domains with $D_{1} \subset \overline{D_{1}} \subset D_{2}$. Let $u$ be $\mathcal{A}$-capacitary potential of $D_{2} \backslash D_{1}$, that is,

$$
\begin{cases}\nabla \cdot \mathcal{A}(\nabla u)=0 & \text { in } D_{2} \backslash \overline{D_{1}}, \\ u=c_{1} & \text { on } \partial D_{1}, \\ u=c_{2} & \text { on } \partial D_{2},\end{cases}
$$


where $c_{1}>c_{2} \geq 0$ are given constants. Then

$$
\limsup _{\substack{z \rightarrow x \\ z \in D_{2} \backslash \overline{D_{1}}}}|\nabla u(z)| \geq \limsup _{\substack{z \rightarrow y_{x} \\ z \in D_{2} \backslash \overline{D_{1}}}}|\nabla u(z)|
$$

for all $x \in \partial D_{1}$.

Proof. Without loss of generality assume that $c_{1}=1$ and $c_{2}=0$ as we may using the the translation and dilation invariance of (1.6). Now let $x \in \partial D_{1}$ and also first assume that $\partial D_{1}$ is not $C^{1}$ at $x$. Note that locally near $x, u$ can be approximated by functions $u^{\epsilon}$, which are solutions to a uniformly elliptic PDE in non-divergence form with ellipticity bounds independent of $\epsilon$ (see [4, section 2.3]). This later fact follows from the structural assumptions on $\mathcal{A}$ as in (i) in Definition 1.1. Then it follows from [33] that there exists a barrier $v$ to such linear equations with $v(x)=u^{\epsilon}(x)=u(x)$, $v \leq u^{\epsilon}$ near $x$ and moreover $|\nabla v(x)|=\infty$. Thus it follows that $|\nabla u(x)|=\infty$. Likewise, if $\partial D_{2}$ is not $C^{1}$ at $y_{x}$, then similarly it follows from [33] that there exists an upper barrier $v$ such that $v\left(y_{x}\right)=u\left(y_{x}\right)$ and $v \geq u$ locally near $y_{x}$ and such that $\left|\nabla v\left(y_{x}\right)\right|=0$. Then it follows that $\left|\nabla u\left(y_{x}\right)\right|=0$, which gives the desired result. Thus in view of the above discussion, we may now restrict our attention to the case when $x, y_{x}$ are in the regular part of $\partial D_{1}$ and $\partial D_{2}$ respectively. Let $x \in \partial D_{1}$ be fixed and let $y_{x}$ be the associated point on $\partial D_{2}$ as described above. Let $P=P_{x, a}$ be a supporting plane at $x$ to $D_{1}$.

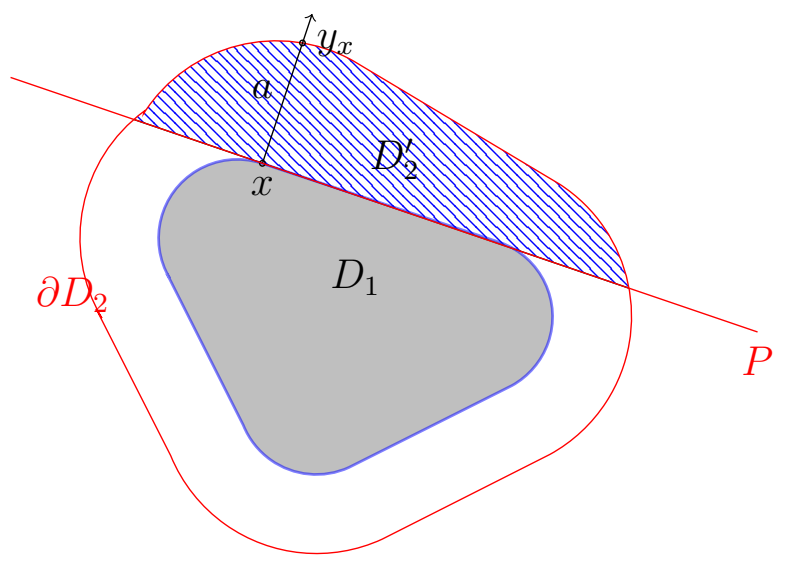

Figure 1. The supporting place $P=P_{x, a}$ and the domains $D_{1}$ and $D_{2}$.

Note that $D_{1} \subset\{P<0\}$ and let $D_{2}^{\prime}:=D_{2} \cap\{P>0\}$. By Lemma 2.3, we may assume that $P=\left\{x_{n}=0\right\}$. Indeed, otherwise after a rotation we have $P=\left\{x_{n}=0\right\}$; we first prove the present lemma for $\tilde{u}$ which is $\tilde{\mathcal{A}}$-harmonic for some $\tilde{\mathcal{A}} \in M_{p}(\alpha)$ and follow by transferring everything back to $u$. Hence assume $P=\left\{x_{n}=0\right\}$ and define $v=u+\alpha x_{n}$, where

$$
\alpha=\limsup _{\substack{z \rightarrow y_{x} \\ z \in D_{2} \backslash \overline{D_{1}}}}|\nabla u(z)|-\epsilon,
$$

with $\epsilon>0$ small. Since $\mathcal{L} u=\mathcal{L} v=0$ in $D_{2}^{\prime}, v$ attains its maximum on $\partial D_{2}^{\prime}$. By the construction of $D_{2}^{\prime}$, the maximum of $v$ is either at $x$ or $y_{x}$. If the maximum were at $y_{x}$, then

$$
0 \leq \limsup _{\substack{z \rightarrow y_{x} \\ z \in D_{2} \backslash D_{1}}} \frac{\partial v}{\partial x_{n}}(z)=-\limsup _{\substack{z \rightarrow y_{x} \\ z \in D_{2} \backslash D_{1}}}|\nabla u(z)|+\alpha=-\epsilon<0 .
$$


It follows that $v$ attains its maximum at $x$, hence

$$
\begin{aligned}
0 \leq & \limsup _{\substack{z \rightarrow x \\
z \in D_{2} \backslash \overline{D_{1}}}} \frac{\partial v}{\partial x_{n}}(z)=\limsup _{\substack{z \rightarrow x \\
z \in D_{2} \backslash \overline{D_{1}}}}|\nabla u(z)|-\alpha \\
& =\limsup _{\substack{z \rightarrow x \\
z \in D_{2} \backslash \overline{D_{1}}}}|\nabla u(z)|-\limsup _{\substack{z \rightarrow y_{x} \\
z \in D_{2} \backslash \overline{D_{1}}}}|\nabla u(z)|+\epsilon .
\end{aligned}
$$

We then conclude the validity of the lemma.

We next show that if $D_{1}$ satisfies the so called the interior ball property as in (1.7) then the $\mathcal{A}$-capacitary function $u$ as above has bounded gradient.

Lemma 3.2. Let $D_{1}, D_{2}$ be as in Lemma 3.1 and let $d_{0}=\min d\left(\partial D_{2}, D_{1}\right)$. Assume also that $D_{1}$ satisfies the interior ball property as in (1.7) with constant $r_{0}$. Then there is a constant $M=M\left(d_{0}, r_{0}, n\right)$ such that

$$
|\nabla u| \leq M \text { in } D_{2} \backslash \overline{D_{1}} \text {. }
$$

Proof. In view of (2.6), it is enough to show that $|\nabla u| \leq M$ on $\partial D_{1} \cup \partial D_{2}$.

We first take care of points on $\partial D_{2}$. Without loss of generality take $c_{1}=1, c_{2}=0$ as (1.6) is invariant under translation and dilation. Let $x \in \partial D_{2}$ be fixed. By rotation, assume, by Lemma 2.3, that $x_{n}=0$ is a supporting hyperplane to $\partial D_{2}$ at $x$ with $D_{2} \subset\left\{x_{n}>0\right\}$ and prove the present lemma for $\tilde{u}$ which is $\tilde{\mathcal{A}}$-harmonic for some $\tilde{\mathcal{A}} \in M_{p}(\alpha)$ and transfer the result back to $u$. Therefore, without loss of generality $x_{n}=0$ is a supporting hyperplane. There exists a supporting hyperplane $x_{n}=d$ to $\partial D_{1}$ for which $D_{1} \subset\left\{x_{n}>d\right\}$. Let $\tilde{D}_{2}=D_{2} \cap\left\{0<x_{n}<d\right\}$ and let $\tilde{u}=x_{n} / d$. Basic comparison principle applied to positive weak solutions of $\mathcal{A}$-harmonic PDEs gives $u \leq \tilde{u}$ in $\tilde{D}_{2}$. This observation and the fact that $u(x)=\tilde{u}(x)$ implies

$$
|\nabla u(x)| \leq|\nabla \tilde{u}(x)| \leq \frac{1}{d} \leq \frac{1}{d_{0}} .
$$

This gives the desired results for points on $\partial D_{2}$.

In order to show the same estimates for points on $\partial D_{1}$, we proceed as follows. We first construct a barrier as we did in the proof of Lemma 2.4 and then we prove that Lemma 3.1 holds for $u_{\epsilon}$. Finally, using Lemmas 2.1 and 2.2 we conclude that Lemma 3.1 holds for $u$ as well.

3.1. A technique of Beurling. In this subsection we give a brief introduction to a technique used by Beurling in [11] and in [25] as well. To this end, recall that $K$ is a convex domain and let

$$
\mathcal{C}:=\left\{\Omega \text { convex bounded open subset of } \mathbf{R}^{n} \text { with } K \subset \Omega\right\} .
$$

Let $u_{\Omega}$ denote the $\mathcal{A}$-capacitary potential for $\Omega \backslash K$ whenever $\Omega \in \mathcal{C}$. Following [26], we also define

$$
\begin{aligned}
\mathcal{G} & :=\left\{\Omega \in \mathcal{C}: \liminf _{y \rightarrow x, y \in \Omega}\left|\nabla u_{\Omega}(y)\right| \geq c \text { for all } x \in \partial \Omega\right\}, \\
\mathcal{G}_{0} & :=\left\{\Omega \in \mathcal{C}: \liminf _{y \rightarrow x, y \in \Omega}\left|\nabla u_{\Omega}(y)\right|>c \text { for all } x \in \partial \Omega\right\}, \\
\mathcal{B} & :=\left\{\Omega \in \mathcal{C}: \limsup _{y \rightarrow x, y \in \Omega}\left|\nabla u_{\Omega}(y)\right| \leq c \text { for all } x \in \partial \Omega\right\} .
\end{aligned}
$$

In the language of Beurling, $\mathcal{G}$ is the collection of "subsolutions" and $\mathcal{B}$ is the collection of "supersolutions". Our aim is to show that $\mathcal{G} \cap \mathcal{B} \neq \emptyset$. To this end, we will make some observations. 
Lemma 3.3. $\mathcal{B}$ is closed under intersection. That is, if $\Omega_{1}, \Omega_{2} \in \mathcal{B}$, then $\Omega_{1} \cap \Omega_{2}$ $\in \mathcal{B}$.

Proof. We will use the comparison principle for non-negative $\mathcal{A}$-harmonic functions. Let $u_{\Omega_{i}}$ for $i=1,2$ be $\mathcal{A}$-capacitary functions for $\Omega_{i} \in \mathcal{B}$. By the comparison principle, we have $u_{\Omega_{1} \cap \Omega_{2}} \leq \min \left\{u_{\Omega_{1}}, u_{\Omega_{2}}\right\}$ in $\left(\Omega_{1} \cap \Omega_{2}\right) \backslash K$. Furthermore, $\partial\left(\Omega_{1} \cap \Omega_{2}\right) \subset \partial \Omega_{1} \cap \partial \Omega_{2}$, hence given $x \in \partial\left(\Omega_{1} \cap \Omega_{2}\right)$ we can assume without loss of generality $x \in \partial \Omega_{1}$. Then $u_{\Omega_{1}}(x)=0=u_{\Omega_{1} \cap \Omega_{2}}(x)$ and thereupon one concludes that

$$
\limsup _{y \rightarrow x, y \in \Omega_{1} \cap \Omega_{2}}\left|\nabla u_{\Omega_{1} \cap \Omega_{2}}(y)\right| \leq \limsup _{y \rightarrow x, y \in \Omega_{1}}\left|\nabla u_{\Omega_{1}}\right| \leq c .
$$

Therefore $\Omega_{1} \cap \Omega_{2} \in \mathcal{B}$. This finishes the proof of Lemma 3.3.

Our next goal is to show the "stability" of $\mathcal{B}$.

Lemma 3.4. Assume $K$ satisfies the interior ball property. Let $\Omega_{1} \supset \Omega_{2} \supset \ldots$ be a decreasing sequence of domains in $\mathcal{B}$. Let

$$
\Omega=\overline{\bigcap_{n} \Omega_{n}}
$$

Assume $\Omega \in \mathcal{C}$. Then $\Omega \in \mathcal{B}$.

Proof. Let $\Omega_{1} \supset \Omega_{2} \supset \ldots$ be a sequence of domains in $\mathcal{B}$ and let $\left\{u_{k}\right\}$ be a sequence of capacitary $\mathcal{A}$-harmonic functions for $\left\{\Omega_{k}\right\}$ respectively. Then $0 \leq$ $u_{k} \leq 1$. Moreover, by Lemmas 2.1, 2.2, and 3.2 it follows that $\left\{u_{k}, \nabla u_{k}\right\}$ converges uniformly on compact subsets of $\Omega \backslash \bar{K}$ to $\{u, \nabla u\}$ where $u$ is a $\mathcal{A}$-harmonic function in $\Omega \backslash K$. The proof that $u$ is indeed the capacitary $\mathcal{A}$-harmonic function for $\Omega \backslash K$ essentially follows from the convergence of $\Omega_{n}$ to $\Omega$ in the Hausdorff distance sense and Lemma 2.4 .

We next show that $\Omega \in \mathcal{B}$. To this end, let $M=\max _{k}\left(\sup \left|\nabla u_{k}\right|\right)<\infty$, by Lemma 3.2. Let $0<\delta_{k}$ be such that $\delta_{k} \rightarrow 0$ as $k \rightarrow \infty$ and

$$
\frac{\left|\nabla u_{k}\right|^{2}-c^{2}}{M^{2}}-\frac{1}{k} \leq 0 \quad \text { on }\left\{u_{k}=\delta_{k}\right\}
$$

Consider

$$
\frac{u_{k}-\delta_{k}}{1-2 \delta_{k}}
$$

which is non-negative in $\left\{u_{k}>\delta_{k}\right\} \backslash\left\{u_{k}<1-\delta_{k}\right\}$ and has zero boundary values on $\left\{u_{k}=\delta_{k}\right\}$. Recall definition (2.4). By (2.6) applied to $\frac{\left|\nabla u_{k}\right|^{2}-c^{2}}{M^{2}}-\frac{1}{k}$,

$$
\mathcal{L}_{u_{k}}\left(\frac{\left|\nabla u_{k}\right|^{2}-c^{2}}{M^{2}}-\frac{1}{k}\right) \geq 0=\mathcal{L}_{u_{k}}\left(\frac{u_{k}-\delta_{k}}{1-2 \delta_{k}}\right)
$$

in $\left\{u_{k}>\delta_{k}\right\} \backslash\left\{u_{k}<1-\delta_{k}\right\}$. On the other hand, on $\left\{u_{k}=\delta_{k}\right\}$ we have

$$
\frac{\left|\nabla u_{k}\right|^{2}-c^{2}}{M^{2}}-\frac{1}{k} \leq 0=\frac{u_{k}-\delta_{k}}{1-2 \delta_{k}} .
$$

Furthermore, on $\left\{u_{k}=1-\delta_{k}\right\} \cap\left\{u_{k} \geq \delta_{k}\right\}$ we have

$$
\frac{\left|\nabla u_{k}\right|^{2}-c^{2}}{M^{2}}-\frac{1}{k} \leq 1=\frac{u_{k}-\delta_{k}}{1-2 \delta_{k}} .
$$

It follows that

$$
\frac{\left|\nabla u_{k}\right|^{2}-c^{2}}{M^{2}}-\frac{1}{k} \leq \frac{u_{k}-\delta_{k}}{1-2 \delta_{k}} \quad \text { in }\left\{u_{k}>\delta_{k}\right\} \backslash\left\{u_{k}<1-\delta_{k}\right\} .
$$


Given $\epsilon>0$ one can find a neighborhood $U_{\epsilon}$ of $\partial \Omega$ such that

$$
u_{k} \leq \epsilon \quad \text { in } U_{\epsilon} .
$$

Letting $k \rightarrow \infty$ and using (3.1) we obtain

$$
\frac{\left|\nabla u_{k}\right|^{2}-c^{2}}{M^{2}}-\frac{1}{k} \rightarrow \frac{|\nabla u|^{2}-c^{2}}{M^{2}} \leq u \leq \epsilon \quad \text { as } k \rightarrow \infty
$$

uniformly on compact subsets of $U_{\epsilon} \cap \Omega$. By letting $\epsilon \rightarrow 0$ we conclude the proof of the Lemma.

As a consequence of Lemma 3.4, we claim that if $\mathcal{G}_{0}$ is not empty and for $\Omega_{0} \in \mathcal{G}_{0}$ the set $\left\{\tilde{\Omega} \in \mathcal{B}: \overline{\Omega_{0}} \subset \tilde{\Omega}\right\}$ is not empty, then there exists a domain $\Omega \in\left\{\tilde{\Omega} \in \mathcal{B}: \overline{\Omega_{0}} \subset\right.$ $\tilde{\Omega}\}$ with the property that

$$
\text { if } \hat{\Omega} \in\left\{\tilde{\Omega} \in \mathcal{B}: \overline{\Omega_{0}} \subset \tilde{\Omega}\right\} \text { and } \hat{\Omega} \subset \Omega \text {, then } \hat{\Omega}=\Omega \text {. }
$$

Such a domain $\Omega$ will be called minimal element in $\left\{\tilde{\Omega} \in \mathcal{B}: \overline{\Omega_{0}} \subset \tilde{\Omega}\right\}$. For simplicity, define

$$
\mathcal{C}_{0}:=\left\{\tilde{\Omega} \in \mathcal{B}: \overline{\Omega_{0}} \subset \tilde{\Omega}\right\}
$$

To prove our claim, let

$$
I=\bigcap_{i} \tilde{\Omega}_{i} \quad \text { where } \tilde{\Omega}_{i} \in \mathcal{C}_{0}
$$

Write $I=\bigcap_{i=1}^{\infty} \tilde{\Omega}_{i}$, with $\tilde{\Omega}_{i} \in \mathcal{C}_{0}$. Let $\Omega_{1}=\tilde{\Omega}_{1}$ and $\Omega_{k+1}=\tilde{\Omega}_{k+1} \cap \Omega_{k}$ for $k=2,3, \ldots$. Then each $\Omega_{k}$ is convex and $\Omega_{k} \in \mathcal{B}$ by Lemma 3.3. Applying Lemma 3.4 to $\left\{\Omega_{n}\right\}$ we conclude that

$$
\Omega=\overline{\bigcap_{n} \Omega_{n}} \in \mathcal{B}
$$

This finishes the proof of our claim.

We proceed by studying the behavior of capacitary $\mathcal{A}$-harmonic functions on extremal points of $\Omega$. To set the stage, let $\Omega$ be the minimal element in $\mathcal{C}_{0}$. A point $x \in \partial \Omega$ is called extremal point if there exists a supporting hyperplane to $\Omega$ touching $\partial \Omega$ at $x$ only. Let $E_{\Omega}$ denote the set of extremal points of $\Omega$.

Lemma 3.5. Let $\Omega$ be a minimal element in the class $\mathcal{C}_{0}$ and let $x \in \overline{E_{\Omega}}$. Then

$$
\limsup _{\substack{y \rightarrow x \\ y \in \Omega}}\left|\nabla u_{\Omega}(y)\right|=c .
$$

Proof. The proof will be a contradiction argument. To this end, suppose there exists $y_{0} \in \overline{E_{\Omega}}$ with

$$
\limsup _{\substack{y \rightarrow y_{0} \\ y \in \Omega}}\left|\nabla u_{\Omega}\left(y_{0}\right)\right|=c(1-4 \tilde{\alpha}) .
$$

for some $\tilde{\alpha}>0$. By the Hölder continuity of $\nabla u_{\Omega}$ there exists a neighborhood $\mathcal{N}$ of $\partial \Omega$ with $y_{0} \in \mathcal{N}$ satisfying that

$$
\left|\nabla u_{\Omega}(x)\right| \leq c(1-\tilde{\alpha}) \quad \text { for every } x \in \mathcal{N} \cap \Omega .
$$

Assume that $y_{0} \in E_{\Omega}$. Otherwise, we may choose a sequence in $E_{\Omega}$ converging to $y_{0}$ with the above property.

Let $d>0$ and let $P_{d}$ be a plane such that $d\left(y_{0}, P_{d}\right)=d$ with $P_{d} \cap \Omega \subset \mathcal{N}$. Notice that without loss of generality, by Lemma 2.3 we may assume that $y_{0}=0$, $P_{d}=\left\{x_{n}=d\right\}$. Otherwise, we rotate our coordinate system, work with $\hat{u}$, which is $\hat{\mathcal{A}}$-harmonic for some $\hat{\mathcal{A}} \in M_{p}(\alpha)$, and at the end transfer everything back to $u_{\Omega}$. 
Hence assume $y_{0}=0, P_{d}=\left\{x_{n}=d\right\}$, let $\epsilon>0$ and define $\Omega_{\epsilon}=\Omega \backslash\left\{x_{n} \leq \epsilon\right\}$. Assume $\epsilon$ is small enough so that $\Omega_{0} \subset \Omega_{\epsilon}$. Let $u_{\epsilon}$ be the $\mathcal{A}$-capacitary function for $\Omega_{\epsilon} \backslash K$. As $u_{\epsilon} \leq u_{\Omega}$ on $\partial \Omega_{\epsilon}$, by the comparison principle for non-negative $\mathcal{A}$-harmonic functions we have

$$
0 \leq u_{\epsilon} \leq u_{\Omega} \text { in } \Omega_{\epsilon}
$$

It follows that we have

$$
\limsup \left|\nabla u_{\epsilon}\right| \leq \limsup \left|\nabla u_{\Omega}\right| \leq c \quad \text { on } \partial \Omega \cap \partial \Omega_{\epsilon} .
$$

At the points where $\partial \Omega \cap \partial \Omega_{\epsilon}$ is not $C^{1}$ we claim that $\left|\nabla u_{\epsilon}\right|=0$. This can be done as in [5, Section 7] by considering $\mathcal{A}(\eta, \delta)$ to obtain a uniformly elliptic equation in divergence form and $v_{\epsilon}^{\delta}$ which is $\mathcal{A}(\eta, \delta)$-harmonic in $\Omega_{\epsilon}$. Once again repeating following [5, Section 7], one concludes that $\left|\nabla v_{\epsilon}^{\delta}\right|=0$ and thereupon letting $\delta \rightarrow 0$ we obtain our claim.

Using (3.3) and (3.4) we have

$$
\max _{P_{d} \cap \Omega} u_{\epsilon} \leq \max _{P_{d} \cap \Omega} u_{\Omega} \leq d \sup _{\left\{0 \leq x_{n} \leq d\right\} \cap \Omega}\left|\nabla u_{\Omega}\right| \leq d c(1-\tilde{\alpha}) .
$$

Let now

and note that

$$
v:=u_{\epsilon}+\frac{d c(1-\tilde{\alpha})}{d-\epsilon}\left(d-x_{n}\right)
$$

$$
\mathcal{L}_{u_{\epsilon}} v=\mathcal{L}_{u_{\epsilon}} u_{\epsilon}=0 \text { in } \Omega_{\epsilon} \cap\left\{x_{n}<d\right\} .
$$

Thereupon we conclude that $v$ takes its maximum on the boundary of $\Omega_{\epsilon} \cap\left\{x_{n}<d\right\}$. Moreover, using (3.6) we obtain

$$
v \leq c(1-\tilde{\alpha}) d \text { on } \partial\left(\Omega_{\epsilon} \cap\left\{x_{n}<d\right\}\right) \text { and } v=d c(1-\tilde{\alpha}) \text { on } P_{\epsilon},
$$

as

$$
\partial\left(\Omega_{\epsilon} \cap\left\{x_{n}<d\right\}\right) \subset P_{d} \cup P_{\epsilon} \cup\left(\partial \Omega \cap\left\{\epsilon<x_{n}<d\right\}\right)
$$

Hence we have

$$
0 \geq \frac{\partial v}{\partial x_{n}}=\left|\nabla u_{\epsilon}\right|-\frac{c(1-\tilde{\alpha}) d}{d-\epsilon} \text { on } P_{\epsilon}
$$

By choosing $\epsilon \leq \tilde{\alpha} d$, we obtain $\left|\nabla u_{\epsilon}\right| \leq c$ on $P_{\epsilon}$. In view of this result and (3.5) we have $\Omega_{\epsilon} \in \mathcal{B}$ and by construction $\Omega_{\epsilon} \subset \Omega$. By (3.2) we conclude that $\Omega=\Omega_{\epsilon}$ which is a contradiction, hence the proof of Lemma 3.5 is complete.

We next observe that if $\Omega$ is a minimal element in the class $\mathcal{C}_{0}$ and let $u_{\Omega}$ be the $\mathcal{A}$-capacitary function for $\Omega \backslash K$ then

$$
\left|\nabla u_{\Omega}(x)\right| \geq c \text { for all } x \in \Omega \backslash K \text {. }
$$

To prove (3.7) we use the fact that for every $0<t<1,\left\{x \in \Omega: u_{\Omega}(x)>t\right\}$ is a convex set due to Lemma 2.5. The conclusion follows by applying Lemma 3.1 to $\left\{x \in \Omega: u_{\Omega}>t\right\}$ and $\Omega$, and using Lemma 3.5.

3.2. Final Proof of Theorem 1.4. We split the proof into two steps, existence of $\Omega$ and uniqueness of $\Omega$.

3.2.1. Existence of $\Omega$. In order to prove Theorem 1.4 we show that there exist domains $\Omega_{0}$ and $\Omega_{1}$ such that $\overline{\Omega_{0}} \in \mathcal{G}_{0}$ and $\Omega_{1} \in \mathcal{B}$ with $\overline{\Omega_{0}} \subset \Omega_{1}$. Then from (3.2) there exists a minimal element $\Omega \in \mathcal{C}_{0}$ and by using (3.7) we have $\Omega \in \mathcal{G}$. In view of the definitions of $\mathcal{G}$ and $\mathcal{B}$, this would allow us to assert existence result of Theorem 1.4. Hence to finish the proof of existence, it remains to show the existence of $\Omega_{0} \in \mathcal{G}_{0}$ and $\Omega_{1} \in \mathcal{B}$ with $\overline{\Omega_{0}} \subset \Omega_{1}$. 
Existence of $\Omega_{1} \in \mathcal{B}$ : For this, choose $R_{0}$ large enough so that $K \subset B_{R_{0}}$. Let $R>R_{0}$ large to be fixed below. Without loss of generality assume $0 \in K$. Let $u_{R}$ be the $\mathcal{A}$-capacitary function for $B(0, R) \backslash K$. Using (b) in Lemma 2.4 we can choose $R$ sufficiently large so that

$$
\left|\nabla u_{R}(x)\right| \leq c_{\star}|x|^{\frac{1-n}{p-1}}=c_{\star} R^{\frac{1-n}{p-1}} \leq c .
$$

Therefore, $\Omega_{1}=B(0, R) \in \mathcal{B}$.

Existence of $\Omega_{0} \in \mathcal{G}_{0}$ : Let $R>0$ be as above and $u_{R}$ be the $\mathcal{A}$-capacitary function for $B(0, R) \backslash K$. We first observe from the smoothness of $B(0, R)$ and Lemma 3.1 that there is a constant $C>0$ and a neighbourhood $U$ of $\partial K$ such that

$$
\left|\nabla u_{R}\right| \geq C \text { in } U \backslash K \text {. }
$$

For a given $t, 0<t<1$, let $\Omega_{t}=\left\{x \in B(0, R): u_{R}(x)>1-t\right\}$. Then the $\mathcal{A}$-capacitary function for $\Omega_{t}$ is

$$
u_{\Omega_{t}}(x)=\frac{u_{R}-(1-t)}{t} .
$$

By choosing $t$ sufficiently small we have on $\partial \Omega_{t}$

$$
\left|\nabla u_{\Omega_{t}}\right|=\frac{\left|\nabla u_{R}\right|}{t} \geq \frac{C}{t} \geq c
$$

Therefore, $\Omega_{0}:=\Omega_{t} \in \mathcal{G}_{0}$ and $\overline{\Omega_{0}} \subset \Omega_{1}$

In view of these two observations and our earlier remarks, the existence of $\Omega$ is done.

3.2.2. Uniqueness of $\Omega$. This will follow from [24], where uniqueness was shown for the Laplace equation, and nonlinear elliptic differential equations satisfying properties (i)-(iv) given below, by using the Lavrentèv principle. In order to make use of this result for nonlinear elliptic equations, one needs to have four conditions (see section 4 in [24]);

(i) The PDE is weakly elliptic and satisfies the comparison principle.

(ii) If $u$ is a solution, then rotations and translations are also solutions to some weakly elliptic PDE satisfying comparison principle.

(iii) $u=x_{n}$ is a solution.

(iv) If $\Omega$ and $K$ are both convex and if $u_{\Omega}$ is the $\mathcal{A}$-capacitary function for $\Omega \backslash K$, then superlevels of $u$ are convex; $\Omega_{t}=\{x \in \Omega: u(x)>t\}$ is convex.

Here (i) in (3.8) follows from the structural assumption on $\mathcal{A}$, (ii) follows from Lemma 2.3. Regarding (iii), it is clear that $u=x_{n}$ is $\mathcal{A}$-harmonic, and (iv) follows from Lemma 2.5.

3.2.3. Proof of $\Omega \in C^{1, \gamma}$. To obtain the $C^{1, \gamma}$ regularity of $\Omega$, one repeats the arguments of Vogel [37], which rely on the machinery of [9] and [10].

Furthermore, it follows from applying the Hodograph transform that if $\mathcal{A} \in$ $C^{\infty}\left(\mathbf{R}^{n} \backslash\{0\}\right)$, then $\partial \Omega \in C^{\infty}$, see $[28,29]$. We notice that an interesting alternative method to obtain higher regularity has recently been done in [15], where the authors prove higher order boundary Harnack estimates. See also $[17,16]$ in the context of thin obstacle problems.

Now the proof of Theorem 1.4 is complete. 


\section{References}

[1] ACKer, A.: Interior free boundary problems for the Laplace equation. - Arch. Rational Mech. Anal. 75:2, 1980/81, 157-168.

[2] ACKer, A.: Uniqueness and monotonicity of solutions for the interior Bernoulli free boundary problem in the convex, $n$-dimensional case. - Nonlinear Anal. 13:12, 1989, 1409-1425.

[3] Acker, A., and R. Meyer: A free boundary problem for the $p$-Laplacian: uniqueness, convexity, and successive approximation of solutions. - Electron. J. Differential Equations 1995:08, 1995, 1-20.

[4] Akman, M.: On the dimension of a certain measure in the plane. - Ann. Acad. Sci. Fenn. Math. 39:1, 2014, 187-209.

[5] Akman, M., J. Gong, J. Hineman, J. Lewis, and A. Vogel: The Brunn-Minkowski inequality and a Minkowski problem for nonlinear capacity. - Mem. Amer. Math. Soc. (to appear).

[6] Akman, M., J. Lewis, O. SaAri, and A. Vogel: The Brunn-Minkowski inequality and a Minkowski problem for $\mathcal{A}$-harmonic Green's function. - Preprint, arXiv:1810.03752, 2018.

[7] Akman, M., J. Lewis, and A. Vogel: On the logarithm of the minimizing integrand for certain variational problems in two dimensions. - Anal. Math. Phys. 2:1, 2012, 79-88.

[8] Akman, M., J. Lewis, and A. Vogel: $\sigma$-finiteness of elliptic measures for quasilinear elliptic PDE in space. - Adv. Math. 309, 2017, 512-557.

[9] Alt, H. W., and L. A. Caffarelli: Existence and regularity for a minimum problem with free boundary. - J. Reine Angew. Math. 325, 1981, 105-144.

[10] Alt, H. W., L. A. Caffarelli, and A. Friedman: A free boundary problem for quasilinear elliptic equations. - Ann. Scuola Norm. Sup. Pisa Cl. Sci. (4) 11:1, 1984, 1-44.

[11] Bennewitz, B.: Non-uniqueness in a free boundary problem. - Rev. Mat. Iberoam. 24:2, 2008, $567-595$.

[12] Beurling, A.: On free boundary problems for the Laplace equation. - In: Seminars on analytic functions I, Institute Advance Studies Seminars 345, 1975, 248-263.

[13] Caffarelli, L. A., and J. Spruck: Convexity properties of solutions to some classical variational problems. - Comm. Partial Differential Equations 7:11, 1982, 1337-1379.

[14] DANiluUk, I. İ.: On integral functionals with a variable domain of integration. - Proc. Steklov Inst. Math. 118, 1972. Translated from the Russian by M. K. Nestell, Amer. Math. Soc., Providence, R. I., 1976.

[15] De Silva, D., and O. SAvin: A note on higher regularity boundary Harnack inequality. Discrete Contin. Dyn. Syst. 35:12, 2014.

[16] De Silva, D., and O. SAvin: $C^{\infty}$ regularity of certain thin free boundaries. - Indiana Univ. Math. J. 64:5, 2015, 1575-1608.

[17] De Silva, D., and O. SAvin: Boundary Harnack estimates in slit domains and applications to thin free boundary problems. - Rev. Mat. Iberoam. 32:3, 2016, 891-912.

[18] Flucher, M.: Variational problems with concentration. - Progr. Nonlinear Differential Equations Appl. 36, Birkhäuser Verlag, Basel, 1999.

[19] Flucher, M., and M. RumpF: Bernoulli's free-boundary problem, qualitative theory and numerical approximation. - J. Reine Angew. Math. 486, 1997, 165-204.

[20] Gabriel, R. M.: A result concerning convex level surfaces of 3-dimensional harmonic functions. - J. London Math. Soc. 32, 1957, 286-294.

[21] Gilbarg, D., and N. S. Trudinger: Elliptic partial differential equations of second order. Classics Math., Springer-Verlag, Berlin, 2001. Reprint of the 1998 edition.

[22] Hamilton, R. S.: The inverse function theorem of Nash and Moser. - Bull. Amer. Math. Soc. (N.S.) $7: 1,1982,65-222$. 
[23] Heinonen, J., T. Kilpeläinen, and O. Martio: Nonlinear potential theory of degenerate elliptic equations. - Dover Publications, Inc., Mineola, NY, 2006. Unabridged republication of the 1993 original.

[24] Henrot, A., and H. Shahgholian: Convexity of free boundaries with Bernoulli type boundary condition. - Nonlinear Anal. 28:5, 1997, 815-823.

[25] Henrot, A., and H. Shahgholian: Existence of classical solutions to a free boundary problem for the $p$-Laplace operator. I. The exterior convex case. - J. Reine Angew. Math. 521, 2000, $85-97$.

[26] Henrot, A., and H. Shahgholian: Existence of classical solutions to a free boundary problem for the $p$-Laplace operator. II. The interior convex case. - Indiana Univ. Math. J. 49:1, $2000,311-323$.

[27] Kawohl, B.: Rearrangements and convexity of level sets in PDE. - Lecture Notes in Math. 1150, Springer-Verlag, Berlin, 1985.

[28] Kinderlehrer, D., and L. Nirenberg: Regularity in free boundary problems. - Ann. Scuola Norm. Sup. Pisa Cl. Sci. 4:2, 1977, 373-391.

[29] Kinderlehrer, D., L. Nirenberg, and J. Spruck: Regularity in elliptic free boundary problems. - J. Anal. Math. 34, 1978, 86-119.

[30] LAVRentÈv, M. A.: Variational methods for boundary value problems for systems of elliptic equations. - P. Noordhoff, Ltd., Groningen, 1963.

[31] Lewis, J. L.: Capacitary functions in convex rings. - Arch. Rational Mech. Anal. 66:3, 1977, $201-224$.

[32] Lewis, J. L., N. Lundström, and K. Nyström: Boundary Harnack inequalities for operators of $p$-Laplace type in Reifenberg flat domains. - In: Perspectives in partial differential equations, harmonic analysis and applications, Proc. Sympos. Pure Math. 79, Amer. Math. Soc., Providence, RI, 229-266, 2008.

[33] Miller, K.: Barriers on cones for uniformly elliptic operators. - Ann. Mat. Pura Appl. (4) 76, 1967.

[34] Serrin, J.: A symmetry problem in potential theory. - Arch. Rational Mech. Anal. 43, 1971, 304-318.

[35] Tepper, D. E.: Free boundary problem. - SIAM J. Math. Anal. 5, 1974, 841-846.

[36] TOlKsdorf, P.: Regularity for a more general class of quasilinear elliptic equations. - J. Differential Equations 51:1, 1984, 126-150.

[37] Vogel, A. L.: Symmetry and regularity for general regions having a solution to certain overdetermined boundary value problems. - Atti Sem. Mat. Fis. Univ. Modena 40:2, 1992, 443-484.

Received 11 November $2019 \bullet$ Accepted 5 September 2020 